\title{
Anomalous Diffusion with an Irreversible Linear Reaction and Sorption-Desorption Process
}

\author{
Maike A. F. dos Santos, ${ }^{1}$ Marcelo K. Lenzi, $^{2}$ and Ervin K. Lenzi ${ }^{1}$ \\ ${ }^{1}$ Departamento de Fisica, Universidade Estadual de Ponta Grossa, Avenida General Carlos Cavalcanti 4748, Ponta Grossa, PR, Brazil \\ ${ }^{2}$ Departamento de Engenharia Qumica, Universidade Federal do Paraná, Av. Cel. Francisco H. dos Santos, \\ 210 Jardim das Americas, 81531-990 Curitiba, PR, Brazil
}

Correspondence should be addressed to Ervin K. Lenzi; ervinklenzi@gmail.com

Received 8 May 2017; Accepted 21 June 2017; Published 25 July 2017

Academic Editor: Alkesh Punjabi

Copyright (c) 2017 Maike A. F. dos Santos et al. This is an open access article distributed under the Creative Commons Attribution License, which permits unrestricted use, distribution, and reproduction in any medium, provided the original work is properly cited.

We investigate the diffusion of two different species in a semi-infinite medium considering the presence of linear reaction terms. The dynamics for these species is governed by fractional diffusion equations. We also consider the presence of an adsorption-desorption boundary condition. The solutions for this system are found in terms of the $H$ function of Fox and by analyzing the behavior of the mean square displacement a rich class of diffusion processes is verified. In this sense, we show how the surface effects modify the bulk dynamics and promote an anomalous diffusion of system.

\section{Introduction}

Recently, a great number experiments on biological, physical, and chemical systems have reported a nonlinear time dependence for the mean square displacement connected to anomalous diffusion. Examples can be found in dynamics of biological cell [1-3], crowding in living cell $[4,5]$, and diffusion in random fractal geometries $[6,7]$. In these situations, $\left\langle(\Delta x)^{2}\right\rangle \sim t^{\alpha}(\alpha>1$ superdiffusion and $\alpha<1$ subdiffusion), in contrast to the usual diffusion characterized by the linear time growing for the mean square displacement; that is, $\left\langle(\Delta x)^{2}\right\rangle \sim t$. A framework usually applied, in these contexts, is the continuous time random walk (CTRW) [8]. It may be characterized by waiting-time distributions with a long-tailed behavior which leads one to the fractional diffusion equations as macroscopic formulation $[9,10]$ for the diffusion process. These equations extend the FokkerPlanck equation [11] and have been successfully applied in several scenarios such as adsorption phenomena [12, 13], diffusion of ions in liquids [14], and diffusion in porous media [15]. Concerning the reaction process in the context of the anomalous diffusion, several works [16-18] have raised the question about the suitable extension when reaction terms are incorporated. One formulation was proposed in [19], where the authors considered the fractional operator only acting on the diffusive term. However, this formulation may result in an unrealistic solution. A similar attempt was made in [20], without success. Other ways of extending the fractional diffusion equation to reaction contexts can be found in [21, 22]. To overcome possible unrealistic scenarios, a mesoscopic formulation based on CTRW was proposed in [23-25] leading us to some satisfactory results and, consequently, working as a guide to incorporate reaction terms in the fractional diffusion equations. In particular, the presence of linear reaction terms [23] yields

$$
\frac{\partial}{\partial t} n(x, t)=\mathscr{K} e^{-k t}{ }_{0} \mathscr{D}_{t}^{1-\alpha} \frac{\partial^{2}}{\partial x^{2}}\left(e^{k t} n(x, t)\right)-k n(x, t),
$$

where $n(x, t)$ represents a density function of a species, $\mathscr{K}$ is the diffusion coefficient, and $k$ is related to the reaction process present in the bulk; ${ }_{0} \mathscr{D}_{t}^{\alpha}(\cdots)$ is Riemann-Liouville fractional derivative [26] defined as follows:

$$
{ }_{0} \mathscr{D}_{t}^{\alpha} f(t)=\frac{1}{\Gamma(\alpha)} \frac{d}{d t} \int_{0}^{t} \frac{d t^{\prime}}{\left(t-t^{\prime}\right)^{1-\alpha}} f\left(t^{\prime}\right) ;
$$

for $0<\alpha<1$ and for $\alpha=1$ the standard form of the differential operator of first-order is recovered. Equation (1) 
was also generalized to the case of $N$ species in [24] by taking into account linear reaction terms, where solutions for the cases with two species in a reversible $1 \rightleftharpoons 2$ and irreversible process $1 \rightarrow 2$ were obtained.

Here, we analyze the solutions of these processes governed by fractional diffusion equations for the two species, in semi-infinite space, that is, $x \geq 0$, with sorption-desorption boundary conditions, where an irreversible process, that is, $1 \rightarrow 2$, is present in the bulk. We consider the following equation:

$$
\frac{\partial}{\partial t} n(x, t)=e_{0}^{R t} \mathscr{D}_{t}^{1-\alpha} \frac{\partial^{2}}{\partial x^{2}}\left(D e^{-R t} n(x, t)\right)+R n(x, t)
$$

subjected to the boundary conditions

$$
\begin{aligned}
\left.e^{-k t} \mathscr{K}_{10} \mathscr{D}_{t}^{1-\alpha} \frac{\partial}{\partial x} e^{k t} n_{1}(x, t)\right|_{x=0} & =-\phi(t), \\
\left.\mathscr{K}_{2} \frac{\partial}{\partial x} n_{2}(x, t)\right|_{x=0} & =0
\end{aligned}
$$

where $\phi(t)$ may represent an sorption-desorption process. In (3), $n^{T}(x, t)=\left(n_{1}(x, t), n_{2}(x, t)\right)$ is related to the numbers of species present in the bulk. $R$ and $D$ are matrix

$$
\begin{aligned}
R & =\left[\begin{array}{cc}
-k & 0 \\
k & 0
\end{array}\right], \\
D & =\left[\begin{array}{cc}
\mathscr{K}_{1} & 0 \\
0 & \mathscr{K}_{2}
\end{array}\right],
\end{aligned}
$$

respectively. It is worth mentioning that results obtained from (3) were used to investigate biological systems [27, 28]. In particular, they have been applied to analyze experimental results of fluorescence recovery after photobleaching (FRAP) in biological systems [29], which is an experimental method widely used to explore binding interactions in cells in vitro and in vivo [30].

The results obtained from (3) subjected to (4) lead us to comprehend how the processes present on the surface and in the bulk (reaction) influence the dynamic of the species present in system. In particular, these results are presented in the next section, Section 2, and show a rich class of processes which can be related to anomalous diffusion. In Section 3, we present our discussion and conclusions.

\section{Subdiffusion and Linear Reaction}

Then, we focus our attention on the solutions of (3) for the two species, by considering that initially in the bulk one of the species is present and the other is absent; for example, $n_{1}(x, t=0)=\delta(x)$ and $n_{2}(x, t=0)=0$. Thus, we may analyze how the reaction process influences the spreading of species 1 and the production of species 2 , as well as the surface effects of both.
After performing some calculations, we can expand (3) in a set of coupled equations as follows:

$$
\begin{aligned}
& \frac{\partial}{\partial t} n_{1}(x, t)=e^{-k t} \mathscr{K}_{10} \mathscr{D}_{t}^{1-\alpha}\left(e^{k t} \frac{\partial^{2}}{\partial x^{2}} n_{1}(x, t)\right) \\
& -k n_{1}(x, t), \\
& \frac{\partial}{\partial t} n_{2}(x, t)=\mathscr{K}_{20} \mathscr{D}_{t}^{1-\alpha}\left(\frac{\partial^{2}}{\partial x^{2}}\left(n_{1}(x, t)+n_{2}(x, t)\right)\right) \\
& +k n_{1}(x, t) \\
& -\mathscr{K}_{1} e^{-k t}{ }_{0} \mathscr{D}_{t}^{1-\alpha}\left(e^{k t} \frac{\partial^{2}}{\partial x^{2}} n_{1}(x, t)\right) \\
& -\left(\mathscr{K}_{2}-\mathscr{K}_{1}\right)_{0} \mathscr{D}_{t}^{1-\alpha}\left(e^{k t} \frac{\partial^{2}}{\partial x^{2}} n_{1}(x, t)\right) .
\end{aligned}
$$

Notice that these equations are coupled by the reaction term: the fact that $k \neq 0$ and $\mathscr{K}_{1}=\mathscr{K}_{2}$ have as a particular case the situation worked out in [24]. This set of equations subjected to the boundary conditions given by (4) can be solved using the Fourier $\left(\mathscr{F}\{f(x, t)\}=\int_{0}^{\infty} d x \cos (\omega x) f(x, t), \mathscr{F}^{-1}\{f(\omega, t)\}=\right.$ $\left.(2 / \pi) \int_{0}^{\infty} d \omega \cos (\omega x) f(\omega, t)\right)$ and Laplace $(\mathscr{L}\{f(x, t)\}=$ $\left.\int_{0}^{\infty} d t e^{-s t} f(x, t), \mathscr{L}^{-1}\{f(x, t)\}=(1 / 2 \pi i) \int_{c-i \infty}^{c+i \infty} d s e^{s t} f(x, t)\right)$ transforms.

Applying these integral transforms in (6), after changing of variables $\widehat{\phi}(t)=\phi(t) e^{k t}, \widehat{n}_{1}(x, t)=n_{1}(x, t) e^{k t}$, and $\Psi(x, t)=$ $n_{1}(x, t)+n_{2}(x, t)$, we obtain

$$
\begin{aligned}
& \Psi(\omega, s)=\frac{1}{s+\mathscr{K}_{2} s^{1-\alpha} \omega^{2}}(\cos (\omega l) \\
& \quad+\left(\mathscr{K}_{2}-\mathscr{K}_{1}\right) s^{1-\alpha} \omega^{2} \widehat{n}_{1}(\omega, s)+\frac{\mathscr{K}_{2} s^{1-\alpha}}{\mathscr{K}_{1}(s+k)^{1-\alpha}} \phi(s) \\
& \left.\quad-\left(\mathscr{K}_{2}-\mathscr{K}_{1}\right) \frac{1}{\mathscr{K}_{1}} \widehat{\phi}(s)\right), \\
& \widehat{n}_{1}(\omega, s)=\frac{1}{s+\mathscr{K}_{1} s^{1-\alpha} \omega^{2}}(\cos (\omega l)+\widehat{\phi}(s))
\end{aligned}
$$

for the initial conditions $n_{1}(x, 0)=\delta(x-l)$ and $n_{2}(x, 0)=0$. Performing the inverse of Fourier transform yields

$$
\begin{aligned}
& \Psi(x, s)=\frac{1}{2 s \sqrt{\mathscr{K}_{2} / s^{\alpha}}} e^{-\sqrt{s^{\alpha} / \mathscr{K}_{2}}|x-l|}+\frac{1}{2 s \sqrt{\mathscr{K}_{2} / s^{\alpha}}} \\
& \cdot e^{-\sqrt{s^{\alpha} / \mathscr{K}_{2}}|x+l|}+\frac{1}{s \sqrt{\mathscr{K}_{2} / s^{\alpha}}} \\
& \cdot e^{-\sqrt{s^{\alpha} / \mathscr{K}_{2}} x}\left(\left(\mathscr{K}_{2}-\mathscr{K}_{1}\right)\left(\frac{1}{\mathscr{K}_{2}}-\frac{1}{\mathscr{K}_{1}} \widehat{\phi}\right)\right. \\
& \left.+\mathscr{K}_{2} s^{1-\alpha} \frac{\phi(s)}{\mathscr{K}_{1}(s+k)^{1-\alpha}}\right)-\int_{0}^{\infty} d x^{\prime} \frac{\mathscr{K}_{2}-\mathscr{K}_{1}}{2 s \mathscr{K}_{2} \sqrt{\mathscr{K}_{2} / s^{\alpha}}} \\
& \cdot(1-s) \widehat{n}_{1}\left(x^{\prime}, s\right)\left(e^{-\sqrt{s^{\alpha} / \mathscr{K}_{2}}\left|x-x^{\prime}\right|}+e^{-\sqrt{s^{\alpha} / \mathscr{K}_{2}}\left|x+x^{\prime}\right|}\right) \text {, }
\end{aligned}
$$




$$
\begin{aligned}
& \widehat{n}_{1}(x, s)=\frac{1}{2 s \sqrt{\mathscr{K}_{2} / s^{\alpha}}}\left(e^{-\sqrt{s^{\alpha} / \mathscr{K}_{2}}|x-l|}+e^{-\sqrt{s^{\alpha} / \mathscr{K}_{2}}|x+l|}\right) \\
& +\frac{1}{s \sqrt{\mathscr{K}_{2} / s^{\alpha}}} e^{-\sqrt{s^{\alpha} / \mathscr{K}_{2}} x} \widehat{\phi}(s) .
\end{aligned}
$$

So, performing the Laplace integral transform, we obtain for species 1

$$
\begin{aligned}
& n_{1}(x, t) \\
& =\frac{e^{-t k}}{\sqrt{4 \mathscr{K}_{1} t^{\alpha}}}\left(H _ { 1 , 1 } ^ { 1 , 0 } \left[\left.\frac{1}{\sqrt{\mathscr{K}_{1} t^{\alpha}}}|x-l|\right|^{(1-(\alpha / 2), \alpha / 2)}(0,1)\right.\right. \\
& \left.+H_{1,1}^{1,0}\left[\left.\frac{1}{\sqrt{\mathscr{K}_{1} t^{\alpha}}}|x+l|\right|^{(1-(\alpha / 2), \alpha / 2)}\right]\right)+\int_{0}^{t} d t^{\prime} \\
& \cdot \frac{e^{-k t^{\prime}}}{\sqrt{\mathscr{K}_{1} t^{\prime \alpha}}} H_{1,1}^{1,0}\left[\left.\frac{1}{\sqrt{\mathscr{K}_{1} t^{\prime \alpha}}}|x|\right|^{(1-(\alpha / 2), \alpha / 2)}(0,1)\right. \\
& \left.-t^{\prime}\right) .
\end{aligned}
$$

For species 2, we may use the equation $n_{2}(x, t)=\Psi(x, t)-$ $n_{1}(x, t)$, where

$$
\begin{aligned}
& \Psi(x, t)=\frac{1}{\sqrt{4 \mathscr{K}_{1} t^{\alpha}}} \\
& \cdot \frac{\mathscr{K}_{1}}{\mathscr{K}_{2}}\left(H_{1,1}^{1,0}\left[\left.\frac{1}{\sqrt{\mathscr{K}_{1} t^{\alpha}}}|x-l|\right|^{(1-(\alpha / 2), \alpha / 2)}\right](0,1)\right] \\
& \left.+H_{1,1}^{1,0}\left[\left.\frac{1}{\sqrt{\mathscr{K}_{1} t^{\alpha}}}|x+l|\right|^{(1-(\alpha / 2), \alpha / 2)}\right]\right)-\int_{0}^{\infty} d x^{\prime} \\
& \cdot \int_{0}^{t} d t^{\prime} \frac{\mathscr{K}_{2}-\mathscr{K}_{1}}{\mathscr{K}_{2} \sqrt{4 \mathscr{K}_{2}\left(t-t^{\prime}\right)^{\alpha}}} \\
& \cdot H_{1,1}^{1,0}\left[\frac{\left|x-x^{\prime}\right|}{\left.\sqrt{\mathscr{K}_{2}\left(t-t^{\prime}\right)^{\alpha}}\right|^{(1-(\alpha / 2), \alpha / 2)}(0,1)}\right]\left(1-\frac{\partial}{\partial t^{\prime}}\right) \\
& \cdot \widehat{n}_{1}\left(x^{\prime}, t^{\prime}\right)-\int_{0}^{\infty} d x^{\prime} \int_{0}^{t} d t^{\prime} \frac{\left(\mathscr{K}_{2}-\mathscr{K}_{1}\right)}{\mathscr{K}_{2} \sqrt{4 \mathscr{K}_{2}\left(t-t^{\prime}\right)^{\alpha}}} \\
& \cdot H_{1,1}^{1,0}\left[\left.\frac{\left|x+x^{\prime}\right|}{\sqrt{\mathscr{K}_{2}\left(t-t^{\prime}\right)^{\alpha}}}\right|_{(1-(\alpha / 2), \alpha / 2)} ^{(0,1)}\right]\left(1-\frac{\partial}{\partial t^{\prime}}\right)
\end{aligned}
$$

$$
\begin{aligned}
& \cdot \widehat{n}_{1}\left(x^{\prime}, t^{\prime}\right)+\int_{0}^{t} \frac{d t^{\prime}}{\sqrt{\mathscr{K}_{2} t^{\prime \alpha}}}
\end{aligned}
$$

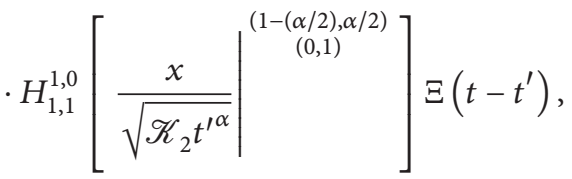

with

$\Xi(t)$

$$
\begin{aligned}
= & \left(\mathscr{K}_{2}-\mathscr{K}_{1}\right)\left(\frac{1}{\mathscr{K}_{2}} \delta(t)-\frac{1}{\mathscr{K}_{1}} e^{k t} \phi(t)\right) \\
& +\frac{1}{\Gamma(1-\alpha)} \frac{\mathscr{K}_{2}}{\mathscr{K}_{1}} \int_{0}^{t} \frac{d t^{\prime}}{\left(t-t^{\prime}\right)^{\alpha}} e^{-k\left(t-t^{\prime}\right)}{ }_{0} \mathscr{D}_{t^{\prime}}^{1-\alpha} \phi\left(t^{\prime}\right) .
\end{aligned}
$$

In (9) and (10), we have the presence of $H$ function of Fox [31],

$$
\begin{aligned}
& H_{p, q}^{m, n}\left[\left.z\right|_{b_{q}, B_{q}} ^{a_{p}, A_{p}}\right]=\frac{1}{2 \pi i} \int_{L} \chi(s) z^{s} d s, \\
& \chi(s)=\frac{\prod_{j=1}^{m} \Gamma\left(b_{j}-B_{j} s\right) \Pi_{j=1}^{m} \Gamma\left(1-a_{j}-A_{j} s\right)}{\prod_{j=1}^{p} \Gamma\left(a_{j}-A_{j} s\right) \Pi_{j=1}^{q} \Gamma\left(1-b_{j}-B_{j} s\right)},
\end{aligned}
$$

with the contour $L$ defined in [8]. It is worth mentioning that the asymptotic behavior of this function in these equations is essentially characterized by stretched exponentials. In fact, after performing some calculations, it is possible to show that

$$
\begin{aligned}
& \frac{1}{\sqrt{4 \mathscr{K}_{1} t^{\alpha}}} H_{1,1}^{1,0}\left[\left.\frac{|x|}{\sqrt{\mathscr{K}_{1} t^{\alpha}}}\right|_{\left(\begin{array}{c}
(1-(\alpha / 2), \alpha / 2) \\
(0,1)
\end{array}\right.} \frac{1}{\sqrt{\pi \mathscr{K}_{1} t^{\alpha}}}\right. \\
& \cdot \sqrt{\frac{1}{2-\alpha}}\left(\frac{2}{\alpha}\right)^{(1-\alpha) /(2-\alpha)}\left(\frac{|x|}{\sqrt{\mathscr{K}_{1} t^{\alpha}}}\right)^{(\alpha-1) /(2-\alpha)} \\
& \times \exp \left(-\frac{2-\alpha}{2}\left(\frac{\alpha}{2}\right)^{\alpha /(2-\alpha)}\left(\frac{|x|}{\sqrt{\mathscr{K}_{1} t^{\alpha}}}\right)^{2 /(2-\alpha)}\right) .
\end{aligned}
$$

By using these results, we may obtain the survival probability $\delta_{1(2)}(t)=\int_{0}^{\infty} d x n_{1(2)}(x, t)$ which is related to the quantity of each species in the bulk. In particular, for species 1 , we have that

$$
\mathcal{S}_{1}(x, t)=e^{-k t}+e^{-k t} \int_{0}^{t} d t^{\prime} e^{k t^{\prime}} \phi\left(t^{\prime}\right)
$$




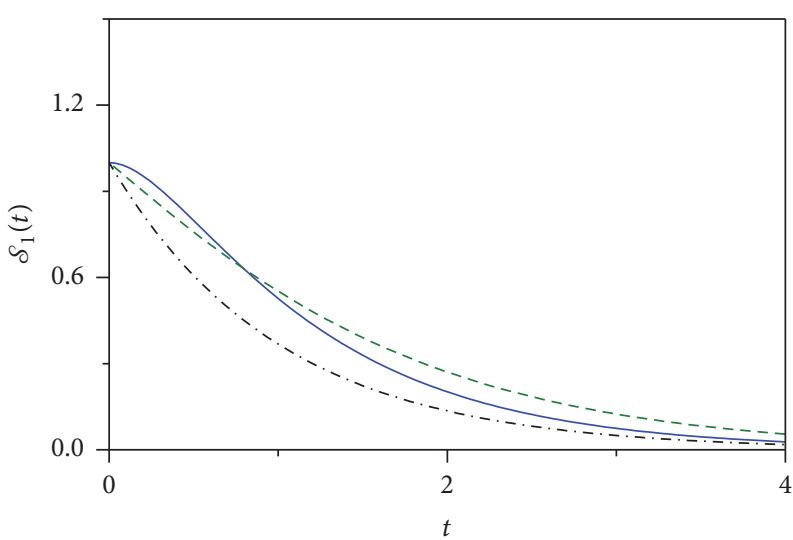

(a)

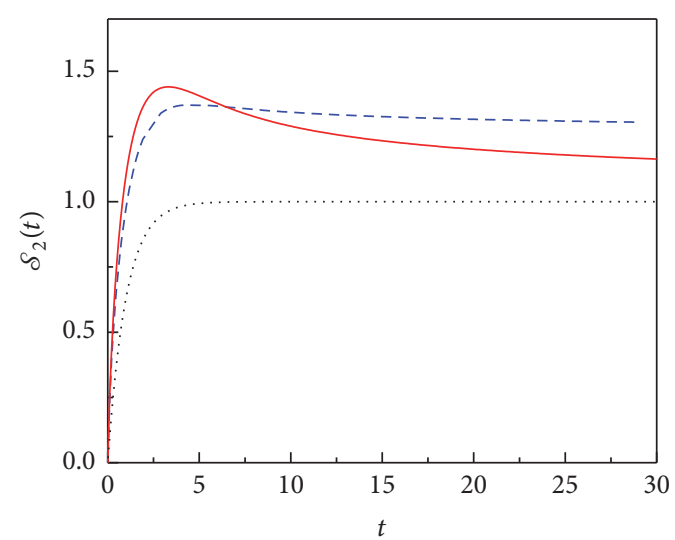

(b)

Figure 1: This figure illustrates the behavior of (14) and (15) versus $t$ for different scenarios. In (a) the blue (solid), black (dashed-dotted), and green (dashed) lines correspond to the cases $\kappa=1$ with $\tau=1 / 3, \kappa=0$ with $\tau=1$, and $\kappa=1 / 2$ with $\tau=1$. In all cases, we have used, for simplicity, $k=1$. In (b) the blue dashed line corresponds to the cases $k=1, \kappa=1, \tau=1 / 3$, and $\mathscr{K}_{1}=2 \mathscr{K}_{2}$. The black dotted line represents the cases $k=1, \kappa=0, \tau=1$, and $\mathscr{K}_{1}=\mathscr{K}_{2}$, respectively. The red solid line is the cases $k=1, \kappa=1 / 2, \tau=1$, and $\mathscr{K}_{1}=\mathscr{K}_{2}$.

and for species 2

$$
\begin{aligned}
& \mathcal{S}_{2}(x, t) \\
& =k \int_{0}^{t} d t^{\prime} \mathcal{S}_{1}\left(x, t^{\prime}\right) \\
& \quad+\frac{1}{\Gamma(1-\alpha)} \frac{\mathscr{K}_{2}}{\mathscr{K}_{1}} \int_{0}^{t} \frac{d t^{\prime}}{\left(t-t^{\prime}\right)^{\alpha}} e^{-k\left(t-t^{\prime}\right)}{ }_{0} \mathscr{D}_{t^{\prime}}^{1-\alpha} \phi\left(t^{\prime}\right) \\
& \quad-\int_{0}^{t} d t^{\prime}\left(1+\left(\mathscr{K}_{2}-\mathscr{K}_{1}\right) \frac{1}{\mathscr{K}_{1}} e^{k t^{\prime}}\right) \phi\left(t^{\prime}\right) .
\end{aligned}
$$

We shall analyze the previous results into a situation characterized by a surface with a variable flux $\phi(t)=\kappa e^{-t / \tau}$ for species 1 . The constant $\kappa$ is related to the rate of the particles through the surface to the bulk and the parameter $\tau$ represents a characteristic time. From these equations, we observe that for $\kappa=0$ the survival probability $\mathcal{S}_{1}(t)$ decreases exponentially; that is, $\mathcal{S}_{1}(t)=e^{-k t}$ with the production of species $2, \mathcal{S}_{2}(t)=1-e^{-k t}$. The cases $\kappa>0$ and $k=$ 0 are characterized by the absence of reaction with a flux through the surface in contact with the bulk. The behavior of $\mathcal{S}_{1}(t)$ and $\mathcal{S}_{2}(t)$ is illustrated in Figure 1 for different scenarios.

Now, we focus our attention on the time dependence manifested by the mean square displacement $(\Delta x)^{2}=\langle(x-$ $\left.\langle x\rangle)^{2}\right\rangle$. Figure 2 shows the behavior of $(\Delta x)^{2}$ for species 1 , when (6) are considered. For initial times, it is characterized by a power-law, $(\Delta x)^{2} \propto t^{\alpha}$, and, for long times, it decreases exponentially. In fact, performing some calculations, it is possible to show for this case that

$$
(\Delta x)_{1}^{2} \sim \frac{2 \mathscr{K}_{1} t^{\alpha} e^{-k t}}{(1-\tau k) \Gamma(\alpha+1)},
$$

in the asymptotic limit of long times. The behavior manifested by species 2 for long times is different from species 1 . It is characterized in the asymptotic limit of $t \rightarrow \infty$ by a powerlaw, $\left\langle(x-\langle x\rangle)^{2}\right\rangle \propto t^{\alpha}$, for $k \neq 0$ (see Figure 3 ). This behavior is essentially the same as the one obtained for the fractional case in absence of reaction term. In both figures (Figures 2 and 3), we also added straight lines to evidence the behaviors manifested during the spreading of the species. In this sense, it is interesting to note that Figure 3(b) shows for the second species three different regimes. In Figure 4, we have shown that the behavior for intermediated times exhibited by the mean square displacement is depending on parameters values. In fact, the difference between the black and the red lines is the value of $k$ which in this case shows a direct influence on the behavior of $(\Delta x)_{2}^{2}$ for intermediate times, before the system reaches the asymptotic behavior.

Another interesting behavior present in these systems is illustrated in Figure 5, where plateaus may be exhibited in some time intervals due to the choice of the boundary conditions considered for species 1 . The plateau for this species indicates that during this time interval the flux by the surface related to species 1 is essentially in equilibrium with the reaction process in the bulk, leading us to a stationary state for the distribution related to this species while the species 2 is produced.

\section{Conclusions}

We have investigated the behavior of a system composed of two species ( 1 and 2 ) which are governed by fractional diffusion equations in a semi-infinity region. In presence of reaction, we have considered a term characterizing an irreversible process and a boundary condition which can 


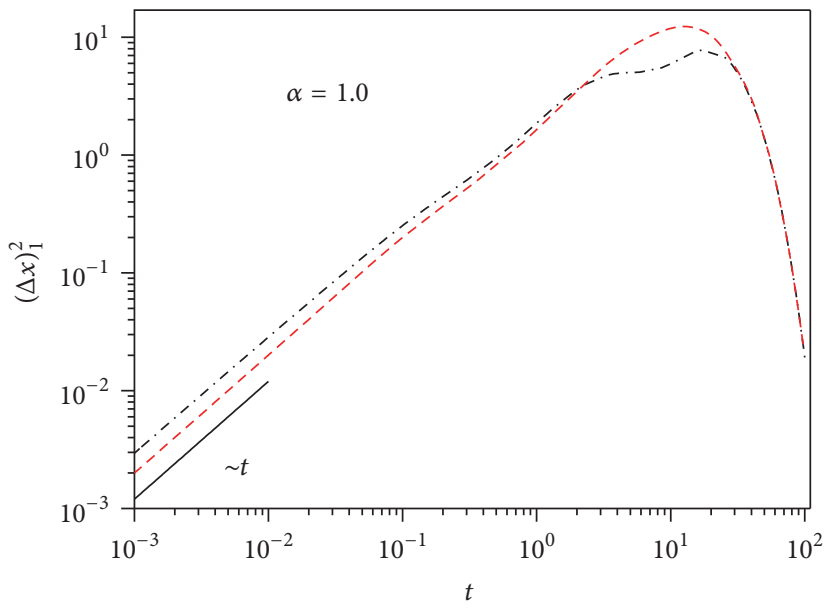

(a)

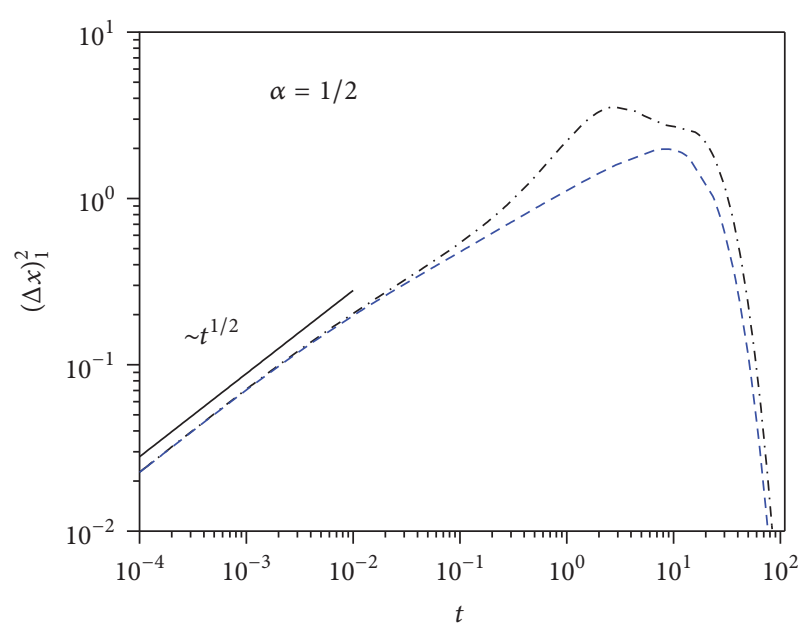

(b)

FIGURE 2: Behavior of the mean square displacement for species 1 versus $t$. In (a) the black dashed-dotted line corresponds to the case $\kappa=1$ with $\tau=1$ and the red dashed line represents the case $\kappa=0$. In (b), the black dashed-dotted line is the cases $\kappa=1$ and $\tau=1$ and the blue dashed line corresponds to the case $\kappa=0$. In all cases, we used $\mathscr{K}_{1}=1$ and $k=0.1$. We also use straight lines to evidence the asymptotic behavior for small times which for $\alpha=1$ is usual and for $\alpha \neq 1$ is anomalous.

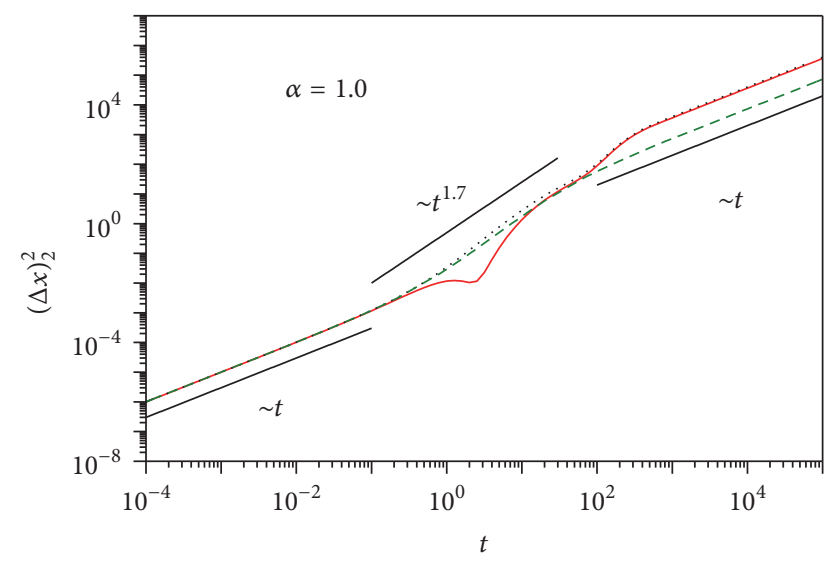

(a)

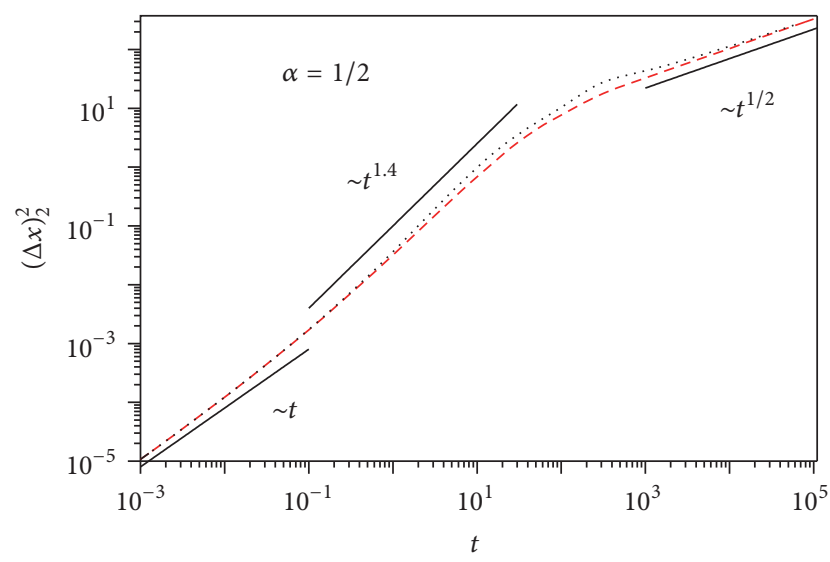

(b)

Figure 3: This figure illustrates the behavior of the mean square displacement for species 2 versus $t$. In (a) the black dotted line corresponds to the cases $\mathscr{K}_{1}=\mathscr{K}_{2}=1, \kappa=1, \tau=1$, and $k=0.01$. The green dashed line is the case $\mathscr{K}_{1}=\mathscr{K}_{2}=1$ with $\kappa=0$ and the red line is the cases $\mathscr{K}_{1}=1, \mathscr{K}_{2}=3.5, \kappa=1$, and $\tau=1$ with $k=0.01$. In (b) the black dotted line is the case $\kappa=1$ and the blue solid line corresponds to $\kappa=0$, respectively. For these cases, we used $\mathscr{K}_{1}=\mathscr{K}_{2}=1, \tau=1$, and $k=10^{-2}$. We also use straight lines to evidence the different behavior of the mean square displacement.

be related to a sorption-desorption process. These equations are coupled by the reaction term which has a direct consequence on the spreading of these species. This point is shown by the behavior of the mean square displacement obtained from (6) for species 1 and 2 which lead us to an anomalous diffusion for both. In particular, for species 2 , we obtained different diffusive behaviors. We have also shown that the intermediated behavior exhibited by the mean square displacement before reaching the asymptotic limit depends on the choice of the parameters present in (3). We have shown the influence of the boundary condition on the system and, in this sense, we also analyzed the behavior of the survival probability for these situations and showed that it depends on the choice of the boundary conditions, that is, $\phi(t)$, which is directly proportional to the flux of particles through the surface.

\section{Conflicts of Interest}

The authors declare that they have no conflicts of interest. 


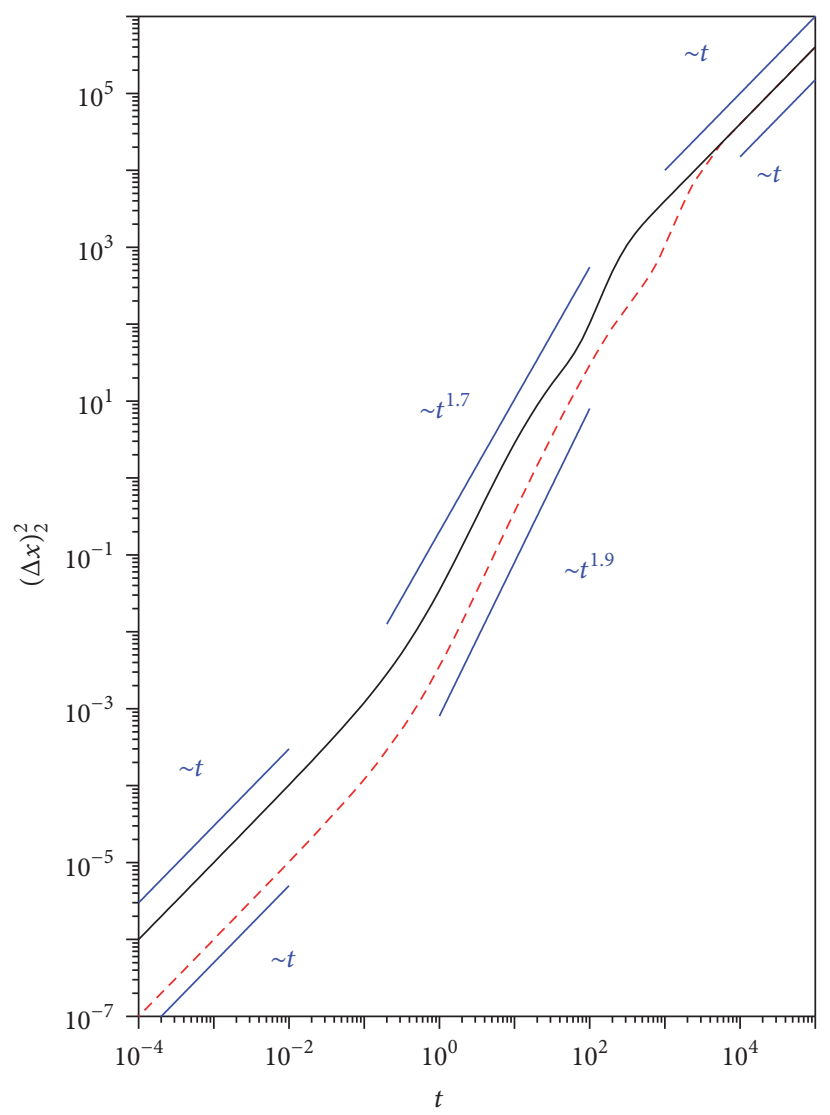

FIGURE 4: This figure illustrates the behavior of the mean square displacement for species 2 versus $t$ for $\alpha=1.0$. The black solid line corresponds to the case $k=10^{-2}$ and the red dashed line is the case $k=10^{-3}$. We consider, for simplicity, $\mathscr{K}_{1}=\mathscr{K}_{2}=1, \kappa=1$, and $\tau=1$. We also use straight lines (blue) to evidence the different behavior of the mean square displacement.

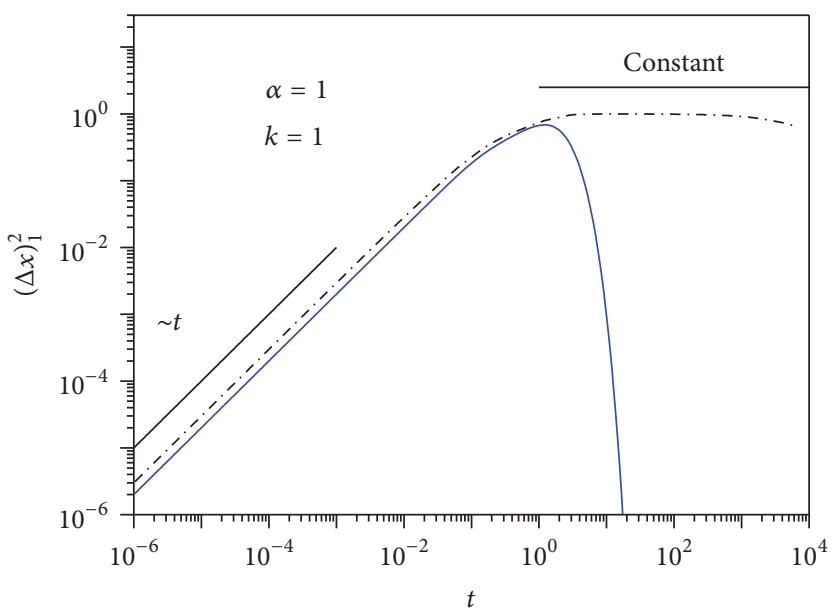

(a)

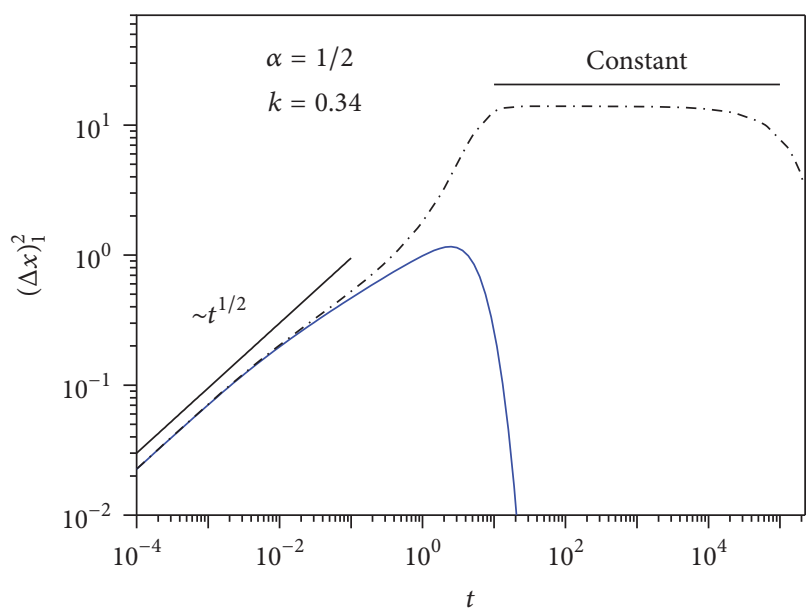

(b)

FIGURE 5: Behavior of the mean square displacement for species 1 versus $t$, when different choices for the boundary conditions are performed. In (a) the black dashed-dotted line corresponds to the case $\kappa=1$ with $\tau=10^{4}$ and the blue solid line considers $\kappa=0$. For these cases, for simplicity, we used $\mathscr{K}_{1}=1$ and $k=1$. In (b) the black dashed-dotted line is the case $\kappa=1$ with $\tau=10^{6}$ and the blue solid line considers $\kappa=0$. We also use straight lines to evidence the different behavior of the mean square displacement. 


\section{Acknowledgments}

The authors thank the CNPq (Brazilian agency) for partial financial support.

\section{References}

[1] F. Höfling and T. Franosch, "Anomalous transport in the crowded world of biological cells," Reports on Progress in Physics, vol. 76, no. 4, Article ID 046602, 2013.

[2] M. J. Saxton, "A biological interpretation of transient anomalous subdiffusion. II. Reaction kinetics," Biophysical Journal, vol. 94, no. 3, pp. 760-771, 2008.

[3] A. J. Ellery, R. E. Baker, S. W. McCue, and M. J. Simpson, "Modeling transport through an environment crowded by a mixture of obstacles of different shapes and sizes," Physica A. Statistical Mechanics and its Applications, vol. 449, pp. 74-84, 2016.

[4] R. Metzler, J.-H. Jeon, and A. G. Cherstvy, "Non-Brownian diffusion in lipid membranes: experiments and simulations," Biochimica et Biophysica Acta - Biomembranes, vol. 1858, no. 10, pp. 2451-2467, 2016.

[5] S. K. Ghosh, A. G. Cherstvy, D. S. Grebenkov, and R. Metzler, "Anomalous, non-Gaussian tracer diffusion in crowded twodimensional environments," New Journal of Physics, vol. 18, no. 1, Article ID 013027, 2016.

[6] Y. Mardoukhi, J.-H. Jeon, and R. Metzler, "Geometry controlled anomalous diffusion in random fractal geometries: looking beyond the infinite cluster," Physical Chemistry Chemical Physics, vol. 17, no. 44, pp. 30134-30147, 2015.

[7] T. Miyaguchi and T. Akimoto, "Anomalous diffusion in a quenched-trap model on fractal lattices," Physical Review E Statistical, Nonlinear, and Soft Matter Physics, vol. 91, no. 1, Article ID 010102, 2015.

[8] R. Metzler and J. Klafter, "The random walk's guide to anomalous diffusion: a fractional dynamics approach," Physics Reports. A Review Section of Physics Letters, vol. 339, no. 1, 77 pages, 2000.

[9] I. M. Sokolov and J. Klafter, "Field-induced dispersion in subdiffusion," Physical Review Letters, vol. 97, no. 14, Article ID 140602, 2006.

[10] R. Metzler and J. Klafter, "The restaurant at the end of the random walk: recent developments in the description of anomalous transport by fractional dynamics," Journal of Physics. A. Mathematical and General, vol. 37, no. 31, pp. R161-R208, 2004.

[11] H. Risken and T. Frank, The FokkerPlanck Equation: Method of Solution and Applications, Springer, 2010.

[12] E. K. Lenzi, L. R. Evangelista, G. Barbero, and F. Mantegazza, "Anomalous diffusion and the adsorption-desorption process in anisotropic media," EPL, vol. 85, no. 2, Article ID 28004, 2009.

[13] G. Barbero and L. R. Evangelista, Adsorption Phenomena and Anchoring Energy in Nematic Liquid Crystals, Taylor and Francis, 2005.

[14] P. A. Santoro, J. L. De Paula, E. K. Lenzi, and L. R. Evangelista, "Anomalous diffusion governed by a fractional diffusion equation and the electrical response of an electrolytic cell," Journal of Chemical Physics, vol. 135, no. 11, Article ID 114704, 2011.

[15] P. Brault, C. Josserand, J.-M. Bauchire, A. Caillard, C. Charles, and R. W. Boswell, "Anomalous diffusion mediated by atom deposition into a porous substrate," Physical Review Letters, vol. 102, no. 4, Article ID 045901, 2009.
[16] K. Seki, M. Wojcik, and M. Tachiya, "Fractional reactiondiffusion equation," Journal of Chemical Physics, vol. 119, no. 4, pp. 2165-2170, 2003.

[17] S. B. Yuste, L. Acedo, and K. Lindenberg, "Reaction front in an reaction-subdiffusion process," Physical Review E: Statistical, Nonlinear, and Soft Matter Physics, vol. 69, no. 3, Article ID 036126, 2004.

[18] T. A. M. Langlands, B. I. Henry, and S. L. Wearne, "Turing pattern formation with fractional diffusion and fractional reactions," Journal of Physics Condensed Matter, vol. 19, no. 6, Article ID 065115, 2007.

[19] B. I. Henry and S. L. Wearne, "Fractional reaction-diffusion," Physica A. Statistical Mechanics and its Applications, vol. 276, no. 3-4, pp. 448-455, 2000.

[20] V. V. Gafiychuk and B. Y. Datsko, "Stability analysis and oscillatory structures in time-fractional reaction-diffusion systems," Physical Review E - Statistical, Nonlinear, and Soft Matter Physics, vol. 75, no. 5, Article ID 055201, 2007.

[21] E. K. Lenzi, M. A. F. Dos Santos, D. S. Vieira, R. S. Zola, and H. V. Ribeiro, "Solutions for a sorption process governed by a fractional diffusion equation," Physica A: Statistical Mechanics and its Applications, vol. 443, pp. 32-41, 2016.

[22] X. Li and M. Xu, "A model for reversible reaction in a subdiffusive regime," Journal of Mathematical Physics, vol. 50, no. 10, Article ID 102708, 102708, 9 pages, 2009.

[23] B. I. Henry, T. A. Langlands, and S. L. Wearne, "Anomalous diffusion with linear reaction dynamics: from continuous time random walks to fractional reaction-diffusion equations," Physical Review E. Statistical, Nonlinear, and Soft Matter Physics, vol. 74, no. 3, Article ID 031116, 2006.

[24] T. A. Langlands, B. I. Henry, and S. L. Wearne, "Anomalous subdiffusion with multispecies linear reaction dynamics," Physical Review E. Statistical, Nonlinear, and Soft Matter Physics, vol. 77, no. 2, Article ID 021111, 2008.

[25] T. A. Langlands and B. I. Henry, "Fractional chemotaxis diffusion equations," Physical Review E. Statistical, Nonlinear, and Soft Matter Physics, vol. 81, no. 5, Article ID 051102, 2010.

[26] I. Podlubny, Fractional Differential Equations, Academic Press, 1999.

[27] E. P. Ipiña and S. P. Dawson, "The effect of reactions on the formation and readout of the gradient of Bicoid," Physical Biology, vol. 14, no. 1, p. 016002, 2017.

[28] M. Kang, E. Di Benedetto, and A. K. Kenworthy, "Proposed correction to Feder's anomalous diffusion frap equations," Biophysical Journal, vol. 100, no. 3, pp. 791-792, 2011.

[29] S. B. Yuste, E. Abad, and K. Lindenberg, "A reactionsubdiffusion model of fluorescence recovery after photobleaching (FRAP)," Journal of Statistical Mechanics: Theory and Experiment, vol. 2014, no. 11, Article ID 11014, 2014.

[30] J. Wu, Y. Fang, V. I. Zarnitsyna, T. P. Tolentino, M. L. Dustin, and C. Zhu, "A coupled diffusion-kinetics model for analysis of contact-area FRAP experiment," Biophysical Journal, vol. 95, no. 2, pp. 910-919, 2008.

[31] A. A. Kilbas, H-Transforms: Theory and Applications, CRC Press, 2004. 


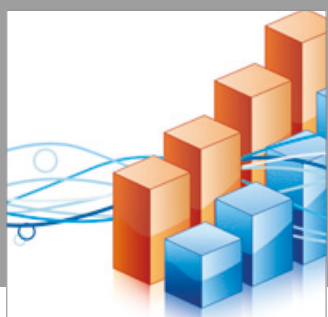

Advances in

Operations Research

vatersals

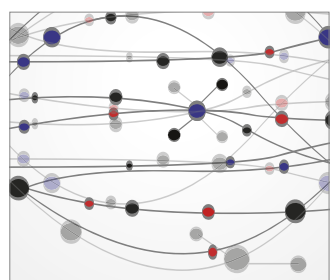

\section{The Scientific} World Journal
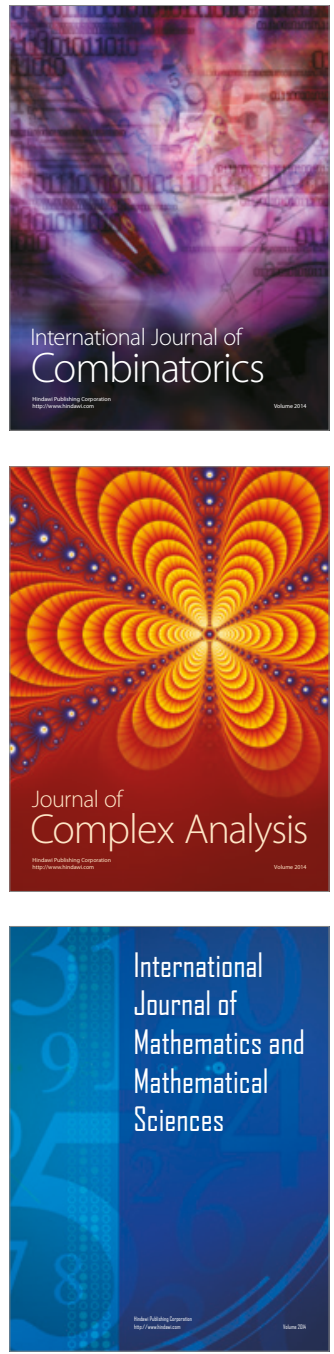
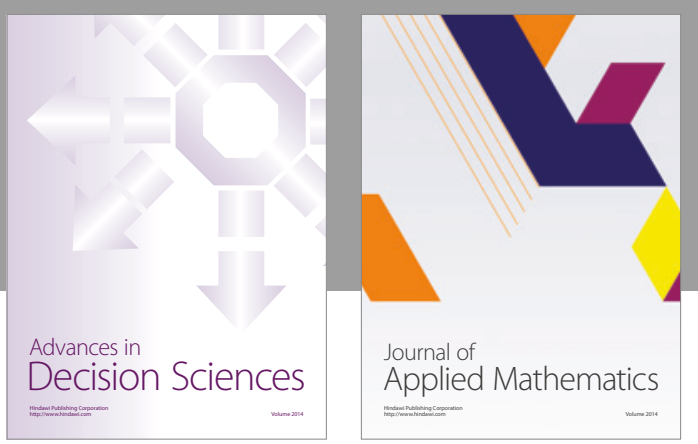

Algebra

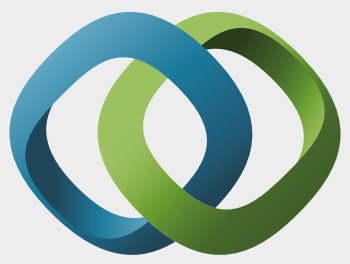

\section{Hindawi}

Submit your manuscripts at

https://www.hindawi.com
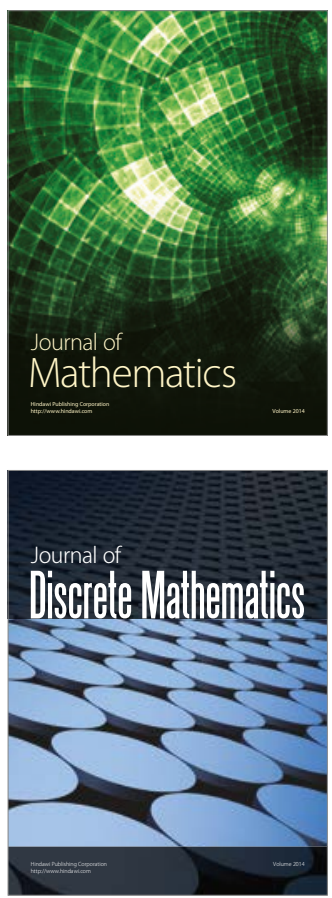

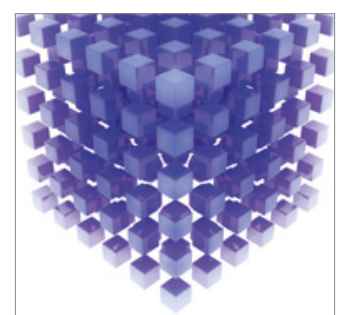

Mathematical Problems in Engineering
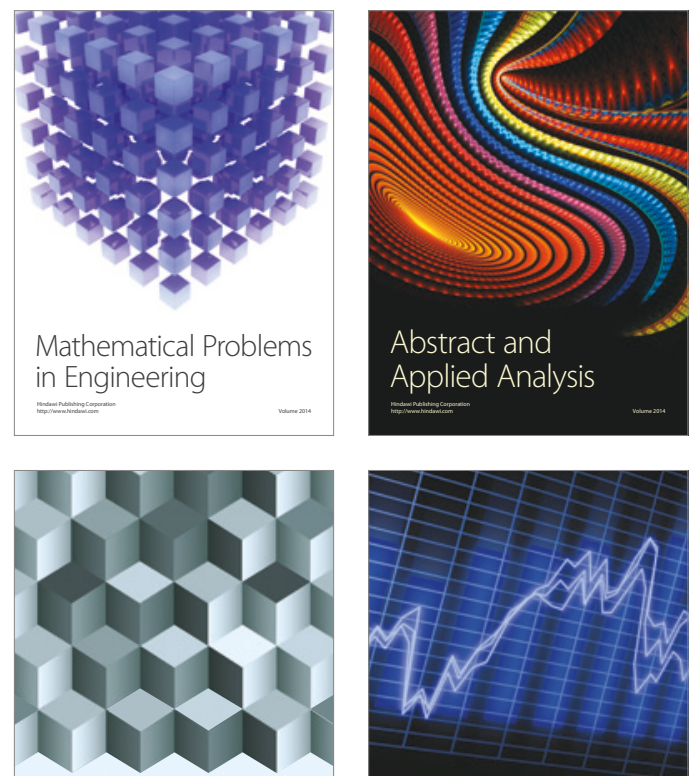

Journal of

Function Spaces

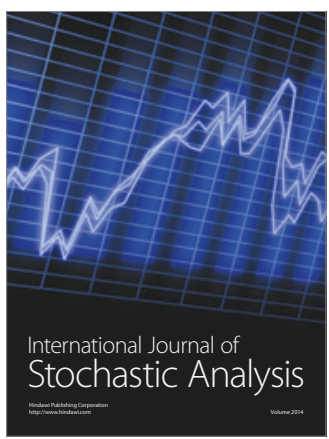

Probability and Statistics
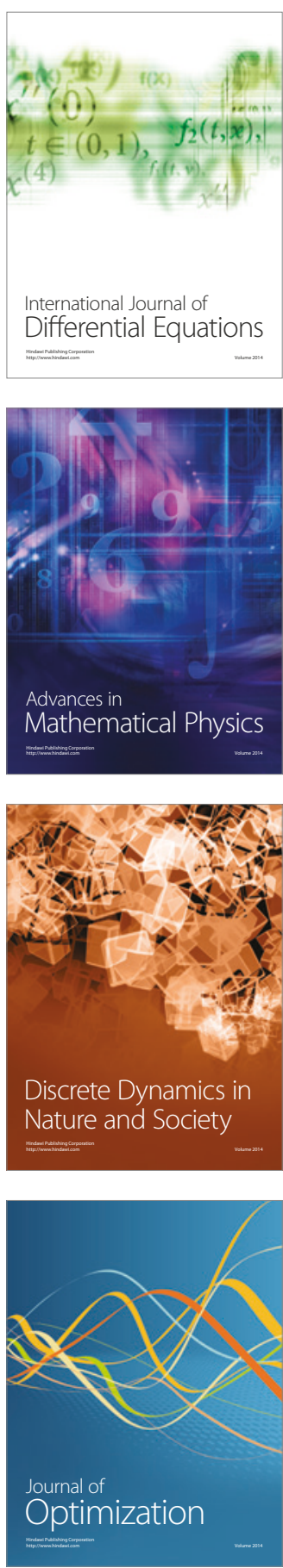